\title{
Plane-stress crack-tip fields for perfectly plastic orthotropic materials
}

\author{
JWO PAN \\ Department of Mechanical Engineering and Applied Mechanics, The University of Michigan, Ann Arbor. \\ MI 48109, USA
}

Received 9 September 1987; accepted 8 April 1988

\begin{abstract}
Plane stress mode I crack-tip fields for perfectly plastic orthotropic materials are studied. Plastic orthotropy is described by Hill's quadratic yield function. The construction of crack-tip fields is based on the general crack-tip field analysis for elastic perfectly plastic materials given by Rice [1] and guided by the corresponding low-hardening power-law solutions. Two very different types of plane-stress crack-tip fields emerge as plastic orthotropy is varied. The first one consists of a centered fan sector in front of the crack tip and two neighboring constant stress sectors. The second one consists of a constant stress sector in front of the crack tip, a constant stress sector bordering the crack face, and a centered fan sector between the two constant stress sectors. All the perfectly plastic crack-tip solutons are verified by the corresponding low-hardening power-law solutions. General trends of crack-tip stress solutions as functions of plastic orthotropy and implications of these solutions to the design of ductile composite materials are discussed.
\end{abstract}

\section{Introduction}

Many engineering materials exhibit anisotropic behavior. To describe plastic anisotropic behavior, Hill $[2,3]$ proposed a phenomenological quadratic yield function for orthotropic materials. Later, Hill [4] proposed non-quadratic yield functions to account for the so-called anomalous behavior of certain materials. To understand the plastic flow near the tip of a crack in an anisotropic material, we adopt Hill's quadratic yield function to describe the plastic flow behavior for its simplicity. We can study the crack-tip behavior using other non-quadratic yield functions in our crack-tip analysis, which is based on the work of Rice [1]. However, our intent is to employ a simple constitutive law that retains certain essential features of plastic anisotropy in order to gain an understanding of the crack-tip behavior as plastic anisotropy is varied.

The asymptotic near-tip solutions for a crack in both power-law hardening and perfectly plastic isotropic materials have been introduced by Hutchinson [5, 6], Rice [7], and Rice and Rosengren [8]. The asymptotic near-tip solutions under combined mode I and II conditions for power-law hardening and perfectly plastic isotropic materials are presented and discussed in Shih [9-11] and for perfectly plastic isotropic materials in Nemat-Nasser and Obata [12]. Hayashi [13] examined the asymptotic near-tip solutions for some power-law hardening materials with mild orthotropy. Pan and Shih $[14,15]$ studied the asymptotic near-tip fields in detail for power-law hardening orthotropic materials under both plane-strain and planestress conditions. For perfectly plastic anisotropic materials, Pan [16] presented the planestrain crack-tip solutions based on the slip-line theory given by Rice [17].

Here, we present mode I plane-stress crack-tip solutions for perfectly plastic orthotropic materials. The solutions are constructed based on different types of assembly of plastic 
sectors as plastic orthotropy is varied. The solutions of different types of crack-tip fields and different sizes of plastic sectors of each type as functions of plastic orthotropy imply and explain some interesting features shown by the corresponding crack-tip solutions for power-law materials [5]. Further, the perfectly plastic crack-tip solutions agree well with the corresponding low-hardening power-law crack-tip solutions [15] and thus represent the low-hardening limit of the corresponding power-law crack-tip solutions.

\section{Governing equations}

In this section, we will follow the development of the governing equations of crack-tip fields for elastic perfectly plastic materials by Rice [1]. We will then use these equations to construct mode I crack-tip fields for perfectly plastic orthotropic materials under plane-stress conditions. Note that the Rice analysis [1] is general and is applicable to anti-plane, plane-strain, and plane-stress problems.

We consider a crack with the crack front parallel to the $x_{3}$ axis in the rectangular Cartesian coordinate system, $x_{1}, x_{2}$, and $x_{3}$, as shown in Fig. 1, where the $x_{3}$ axis is perpendicular to the $x_{1}-x_{2}$ plane. Let $r$ and $\theta$ denote the polar coordinates centered at the crack tip, $\mathbf{e}$ the unit vector in the radial direction, and $\mathbf{h}$ the unit vector in the transverse direction. Note that

$$
\partial r / \partial x_{i}=e_{i}, \quad \partial \theta / \partial x_{i}=h_{i} / r
$$

where the subscript $i$ has a range of 1,2 , and 3 and

$$
e_{1}=h_{2}=\cos \theta, \quad e_{2}=-h_{1}=\sin \theta, \quad e_{3}=h_{3}=0 .
$$

\subsection{Equilibrium}

Within the context of the small strain approach, the equilibrium equations are

$$
\partial \sigma_{i j} / \partial x_{i}+f_{j}=0
$$

where $\sigma_{i j}\left(=\sigma_{j i}\right)$ is the stress tensor, $f_{j}$ is the body force, and the subscripts $i$ and $j$ have a range of 1,2 , and 3 and follow the summation convention. The equilibrium equations (2.3), using

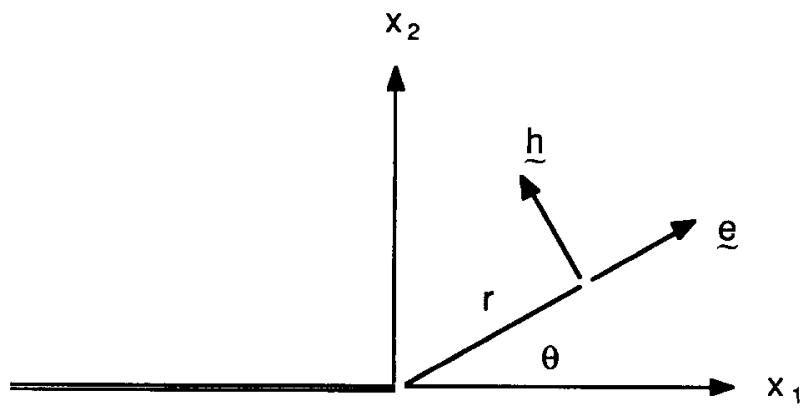

Fig. 1. Conventions at the crack tip. 
the relation $(2.1)$, become

$$
\left(\partial \sigma_{i j} / \partial \theta\right)\left(h_{i} / r\right)+\left(\partial \sigma_{i j} / \partial r\right) e_{i}+f_{j}=0
$$

Since $r f_{j}$ and $r \partial \sigma_{i j} / \partial r \rightarrow 0$ as $r \rightarrow 0$ for perfectly plastic materials [1], the equilibrium equations $(2.3)$ reduce to

$$
h_{i} \sigma_{i j}^{\prime}=0 \text { where } \sigma_{i j}^{\prime}=\left[\partial \sigma_{i j}(r, \theta) / \partial \theta\right]_{r=0}
$$

as $r \rightarrow 0$. Equation (2.4) can be expressed fully in the Cartesian coordinate as

$$
e_{2} \sigma_{11}^{\prime}=e_{1} \sigma_{21}^{\prime}, \quad e_{2} \sigma_{12}^{\prime}=e_{1} \sigma_{22}^{\prime}, \quad e_{2} \sigma_{13}^{\prime}=e_{1} \sigma_{23}^{\prime}
$$

or in the polar coordinate as

$$
\sigma_{r r}-\sigma_{\theta \theta}+\sigma_{r \theta}^{\prime}=0,2 \sigma_{r \theta}+\sigma_{\theta \theta}^{\prime}=0, \sigma_{r 3}+\sigma_{\theta 3}^{\prime}=0 .
$$

For an arbitrary tensor $H_{i j}$, Rice [1] gives the following equality using the equilibrium equations (2.5):

$$
\sigma_{i j}^{\prime} H_{i j}=\left(\sigma_{11}^{\prime}+\sigma_{22}^{\prime}\right) H_{r r}+\sigma_{33}^{\prime} H_{33}+2\left(\sigma_{13}^{\prime} / e_{1}\right) H_{r 3}
$$

\subsection{Yield condition}

The yield condition for perfectly plastic materials can be assumed to have the form

$$
f\left(\sigma_{i j}\right)=0
$$

When the yield surface in the stress space is smooth, the differential form of the yield condition (2.8) can be written as

$$
P_{i j} \sigma_{i j}^{\prime}=0
$$

as $r \rightarrow 0$, where

$$
P_{i j}=\partial f / \partial \sigma_{i j}
$$

Here, $P_{i j}$ represents a symmetric tensor in the direction of the outward normal to the yield surface in the stress space.

The differential yield condition (2.9) together with (2.7), which is obtained using the equilibrium equations $(2.5)$, gives

$$
\left(\sigma_{11}^{\prime}+\sigma_{22}^{\prime}\right) P_{r r}+\sigma_{33}^{\prime} P_{33}+2\left(\sigma_{13}^{\prime} / e_{1}\right) P_{r 3}=0
$$




\subsection{Plane stress plastic sectors}

For two-dimensional plane-stress problems, we can set $\sigma_{31}=\sigma_{32}=\sigma_{33}=0$. The equilibrium equations are

$$
e_{2} \sigma_{11}^{\prime}=e_{1} \sigma_{21}^{\prime}, \quad e_{2} \sigma_{12}^{\prime}=e_{1} \sigma_{22}^{\prime}
$$

These equations in the polar coordinate are

$$
\sigma_{r r}-\sigma_{0 \theta}+\sigma_{r \theta}^{\prime}=0,2 \sigma_{r \theta}+\sigma_{\theta \theta}^{\prime}=0 \text {. }
$$

Equation (2.11), which represents the differential yield condition and the equilibrium condition, simplifies to

$$
\left(\sigma_{11}^{\prime}+\sigma_{22}^{\prime}\right) P_{r r}=0 .
$$

There are three equations, (2.14) and (2.13) (or (2.12)), for the three stresses $\sigma_{11}, \sigma_{12}\left(=\sigma_{21}\right)$, and $\sigma_{22}$. This is a statically determinate problem with two possible solutions:

(1) Constant stress sectors: $\sigma_{11}=$ constant, $\sigma_{12}=\sigma_{21}=$ constant, and $\sigma_{22}=$ constant.

(2) Centered fan sectors: From the viewpoint of the rigid perfectly plastic plane-stress equations, the vanishing extensional components of $P_{i j}$ define the characteristics; therefore, $P_{r r}=0$ defines a fan of radial characteristics.

Under anti-plane and plane-strain conditions, the governing equations and their implications on the crack-tip fields can be found in Rice [1].

\section{Orthotropic plasticity}

We consider the cases where the pronounced preferred crystallographic orientation is already firmly established and restrict the discussion to a range of plastic straining such that further change of plastic orthotropy is negligible. We choose the axes of orthotropic symmetry of a material, $X_{1}, X_{2}$, and $X_{3}$, to coincide with the axes of the Cartesian coordinate system, $x_{1}, x_{2}$, and $x_{3}$, as shown in Fig. 1. Hill's yield condition [2, 3] for orthotropic materials can be expressed in the form

$$
\begin{aligned}
f\left(\sigma_{i j}\right)= & F\left(\sigma_{22}-\sigma_{33}\right)^{2}+G\left(\sigma_{33}-\sigma_{11}\right)^{2}+H\left(\sigma_{11}-\sigma_{22}\right)^{2} \\
& +2 L \sigma_{23}^{2}+2 M \sigma_{31}^{2}+2 N \sigma_{12}^{2}-Q^{2}=0,
\end{aligned}
$$

where $f\left(\sigma_{i j}\right)$ represents the current yield surface in the stress space and $F, G, H, L, M$, and $N$ are the dimensionless constants that describe the state of plastic orthotropy. An effective yield strength parameter $Q$ can be defined to represent the characteristic size of the elastic domain in the stress space. For perfectly plastic materials, $Q$ is a constant. We can set $F=G=H=L / 3=M / 3=N / 3=1 / 6$ and (3.1) becomes Von Mises' yield condition for isotropic materials. 
Let $X_{0}, Y_{0}$, and $Z_{0}$ be the yield stresses along the orthotropic symmetry axes and $R_{0}, S_{0}$, and $T_{0}$ be the shear yield stresses with respect to the orthotropic symmetry axes. As in Pan and Shih $[14,15]$, we identify $Q$ with the shear yield stress $T$. With this normalization, these relations follow (Hill $[2,3]$ ):

$$
\begin{aligned}
G+H & =\left(T_{0} / X_{0}\right)^{2}, \quad H+F=\left(T_{0} / Y_{0}\right)^{2}, \quad F+G=\left(T_{0} / Z_{0}\right)^{2} \\
2 L & =\left(T_{0} / R_{0}\right)^{2}, \quad 2 M=\left(T_{0} / S_{0}\right)^{2}, \quad 2 N=1
\end{aligned}
$$

We introduce the generalized shear and tensile yield stresses, $\tau_{e}$ and $\sigma_{e}$, which are defined by

$$
\begin{aligned}
\tau_{e}^{2}= & \sigma_{e}^{2} / 3=F\left(\sigma_{22}-\sigma_{33}\right)^{2}+G\left(\sigma_{33}-\sigma_{22}\right)^{2}+H\left(\sigma_{11}-\sigma_{22}\right)^{2} \\
& +2 L \sigma_{23}^{2}+2 M \sigma_{31}^{2}+2 N \sigma_{12}^{2}=Q^{2}
\end{aligned}
$$

Invoking the plane-stress condition $\left(\sigma_{13}=\sigma_{23}=\sigma_{33}=0\right)$ and $2 N=1$, the yield condition becomes

$$
f=F \sigma_{22}^{2}+G \sigma_{11}^{2}+H\left(\sigma_{11}-\sigma_{22}\right)^{2}+\sigma_{12}^{2}-\tau_{e}^{2}=0
$$

The yield condition can be expressed in the polar coordinate by stress transformation:

$$
\begin{aligned}
f= & F\left(\sigma_{r r} \sin ^{2} \theta+\sigma_{\theta \theta} \cos ^{2} \theta+\sigma_{r 0} \sin 2 \theta\right)^{2} \\
& +G\left(\sigma_{r r} \cos ^{2} \theta+\sigma_{\theta \theta} \sin ^{2} \theta-\sigma_{r 0} \sin 2 \theta\right)^{2} \\
& +H\left[\left(\sigma_{r r}-\sigma_{\theta \theta}\right) \cos 2 \theta-2 \sigma_{r 0} \sin 2 \theta\right]^{2} \\
& +(1 / 4)\left[\left(\sigma_{r r}-\sigma_{\theta 0}\right) \sin 2 \theta+2 \sigma_{r 0} \cos 2 \theta\right]^{2}-\tau_{e}^{2}=0 .
\end{aligned}
$$

As discussed earlier in Section 2.3, there are two types of plastic sectors: one is the constant stress sector and the other is the centered fan sector. In the centered fan sector, $P_{r}$ vanishes. $P_{r r}$ can be expressed in the polar coordinate as

$$
\begin{aligned}
P_{r r}= & \partial f / \partial \sigma_{r r}=0 \\
= & \left(\sin ^{2} \theta\right) F\left(\sigma_{r r} \sin ^{2} \theta+\sigma_{\theta \theta} \cos ^{2} \theta+\sigma_{r \theta} \sin 2 \theta\right) \\
& +\left(\cos ^{2} \theta\right) G\left(\sigma_{r r} \cos ^{2} \theta+\sigma_{\theta 0} \sin ^{2} \theta-\sigma_{r \theta} \sin 2 \theta\right) \\
& +(\cos 2 \theta) H\left[\left(\sigma_{r r}-\sigma_{\theta \theta}\right) \cos 2 \theta-2 \sigma_{r \theta} \sin 2 \theta\right] \\
& +(\sin 2 \theta)(1 / 4)\left[\left(\sigma_{r r}-\sigma_{\theta \theta}\right) \sin 2 \theta+2 \sigma_{r \theta} \cos 2 \theta\right]
\end{aligned}
$$

When the normality flow law is assumed, the condition $P_{r r}=0$ specifies a rather strong kinematic condition: the plastic strain rate in the radial direction must vanish. 


\section{Mode I crack-tip fields}

Under plane-stress conditions, the crack-tip fields are functions of three material orthotropy parameters: $F, G$, and $H$ (see the yield condition (3.4) or (3.5)). Since we assume that plastic orthotropy remains unchanged under deformation, the crack-tip fields therefore can be expressed as functions of the relative magnitudes of the yield stresses $X_{0}, Y_{0}, Z_{0}$, and $T_{0}$ (see (3.2)). We vary the relative magnitudes of the yield stresses to examine the effects of plastic orthotropy on the crack-tip fields. We only examine four simple cases as in [15].

In this paper, we study mode I symmetric cases only. For the four cases [15] that we consider here, we found that there are two types of crack-tip fields. The first one, a type-A field, shown in Fig. 2(a), consists of a centered fan sector in front of the crack tip and two neighboring constant stress sectors; a discontinuity of the radial stress appears along the border OB of the two constant stress sectors. The other, a type-B field, shown in Fig. 2(b), consists of a constant stress sector in front of the crack tip and a constant stress sector bordering the stress-free crack face. Between the two constant stress sectors, there is a centered fan sector. The assembly of plastic sectors of type-B fields is similar to that of the plane-strain mode I crack-tip fields $[1,7,16,18]$.

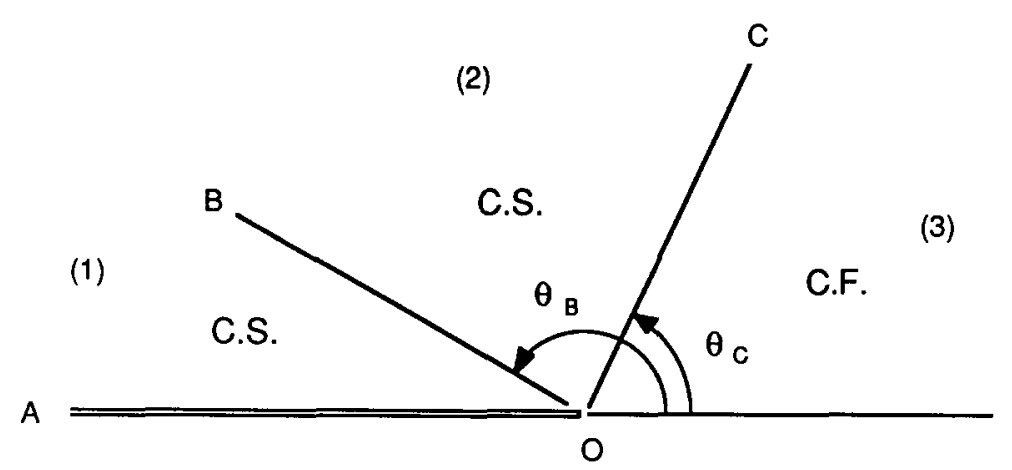

(a)

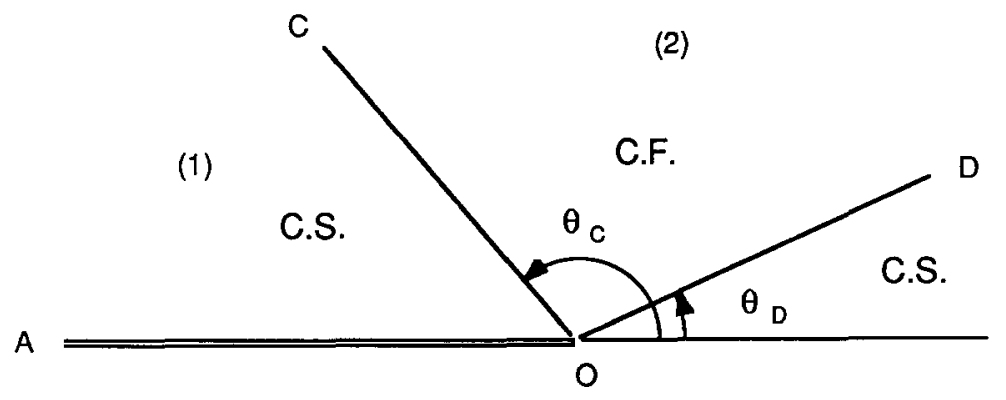

(3)

(b)

Fig. 2. Two possible plane-stress mode I crack-tip fields (C.S. represents a constant stress sector and C.F. represents a centered fan sector). 
In constructing the crack-tip fields, we use the low-hardening solutions of the crack-tip stress fields for power-law materials (Pan and Shih [15]) to guide the selection of either a type-A or a type-B field. We integrate the governing equations of (2.13) and the differentiation of (3.6) by the fourth-fifth Runge-Kutta scheme with error and step-size control. We employ two-parameter shooting methods to meet the symmetry and boundary conditions of the crack-tip fields shown in either Fig. 2(a) or 2(b).

Due to the symmetry conditions, we only need to consider the crack-tip field from $\theta=0^{\circ}$ to $\theta=180^{\circ}$. We reinforce the symmetry conditions such that $\sigma_{r \theta}=0$ at $\theta=0^{\circ}$. To satisfy the stress-free boundary conditions, we have $\sigma_{r \theta}=\sigma_{(j)}=0$ at $\theta=180^{\circ}$. We also reinforce the traction continuity conditions such that $\sigma_{r \theta}$ and $\sigma_{\theta \theta}$ are continuous along the borders of the two sectors of the crack-tip fields.

\subsection{Case I}

First, we examine the case where the strength in the $x_{1}$ direction is increased while the strengths in the other directions remain unchanged, as for isotropic materials, i.e., $X_{0} / Y_{0}>1$ and $Y_{0}=Z_{0}=\sqrt{3} T_{0}$. Figure 3(a) shows the crack-tip stress field for isotropic materials ( $X_{0}=Y_{0}=Z_{0}=\sqrt{3} T_{0}$ ). This is identical to the stress field (a type-A field) presented by Hutchinson [6]. Note that for the perfectly plastic crack-tip stress solutions shown in this paper, the crack-tip stresses are normalized by $\sigma_{\bullet}$. Figures $3(\mathrm{~b})$ and $3(\mathrm{c})$ show the crack-tip stress fields (with $Y_{0}=Z_{0}=\sqrt{3} T_{0}$ ) for $X_{0} / Y_{0}=2$ and 4 , respectively.

All the crack-tip fields shown in Fig. 3 are type-A fields. For isotropic materials, $\theta_{C}$ and $\theta_{B}$, as defined in Fig. 2(a), are calculated as $79.8 \mathrm{deg}$ and $151.2 \mathrm{deg}$, respectively. These values agree with Hutchinson's results [6]. When $X_{0} / Y_{0}$ is increased from 1 to $4, \theta_{C}$ and $\theta_{B}$ increases from 79.8 and 151.2 degs to 86.4 and 170.8 degs, respectively; the discontinuity of the radial stress at the border $\mathrm{OB}$ increases from $1.31 \sigma_{e}$ to $3.63 \sigma_{\rho}$. As shown in Fig. 3, the angular variation of $\sigma_{r r}$ peaks at $\theta=0^{\circ}$ as the strength in the $x_{1}$ direction is increased.

Pan and Shih [15] studied the crack-tip field for power-law hardening orthotropic materials. According to their results, the angular variations of the dimensionless singular crack-tip stress fields, $\tilde{\sigma}_{i j}$ and $\tilde{\sigma}_{e}$, for $n=20$ are shown in Figs. 4(a), 4(b), and 4(c) for the three materials corresponding to Figs. 3(a), 3(b), and 3(c), respectively. Note that $n=20$ represents a stress-strain relation very close to the perfectly plastic stress-strain law and that the angular functions are normalized by setting the maximum value of the $\theta$-variation of $\tilde{\sigma}_{e}$ to unity.

In Fig. 4, the power-law solutions show the same trend as the perfectly plastic solutions in Fig. 3 when the strength in the $x_{1}$ direction is increased. For example, $\tilde{\sigma}_{r r}$ peaks at $\theta=0^{\circ}$ and the magnitude of the apparent discontinuity of $\tilde{\sigma}_{r r}$ near the stress-free crack face increases. As discussed in [15], the increase of strength in the $x_{1}$ direction does not affect the crack-tip stress field very much except the normal stress near the $x_{1}$ direction. This fact is shown in Figs. 3 and 4, which demonstrate that as the strength in the $x_{1}$ direction is increased, $\tilde{\sigma}_{r r}$ increases near the angles $\theta=0^{\circ}$ and $\theta=180^{\circ}$.

The power-law solutions resemble the perfectly plastic solutions in every way except that the power-law solutions are smoother than the perfectly plastic solutions. Note that when we construct the power-law solutions, we assume that the second derivatives of the singular stresses with respect to $\theta$ exist. However, when we construct the perfectly plastic solutions, we assemble these sectors as shown in Fig. 2(a) and we impose the traction continuity 


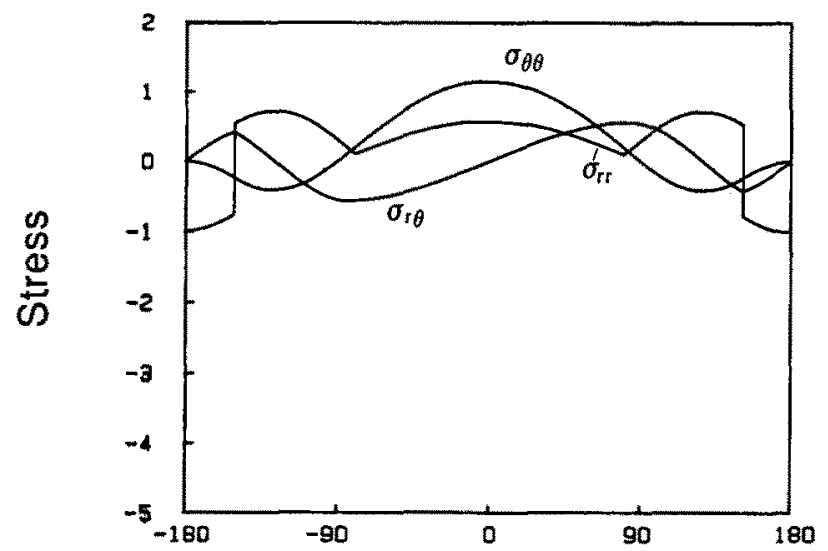

(a)

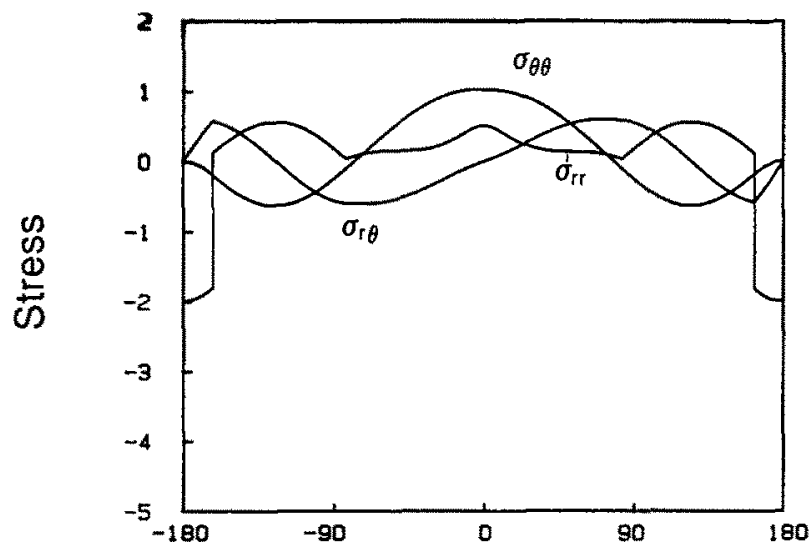

(b)

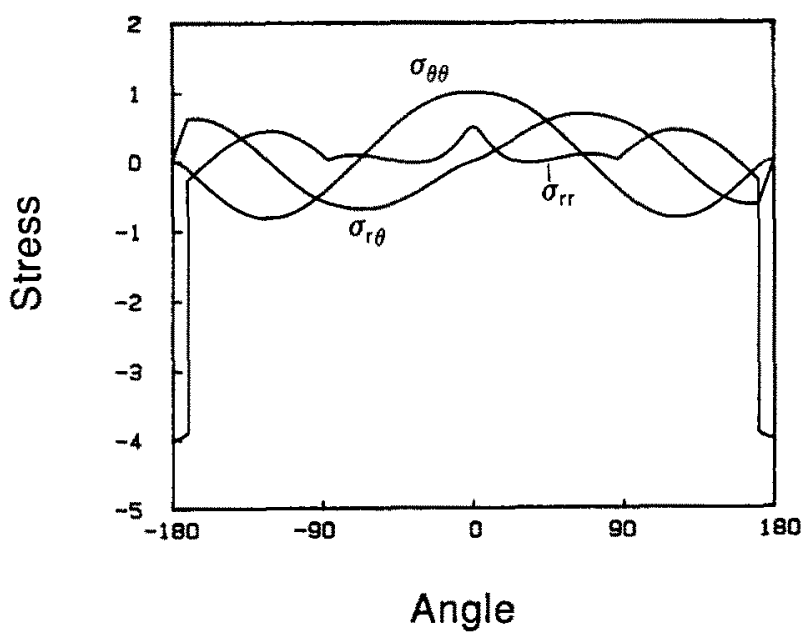

(c)

Fig. 3. Case 1: Angular distributions of the perfectly plastic crack-tip stress fields for $Y_{0}=Z_{0}=\sqrt{3} T_{0}$ and $X_{0} / Y_{0}=$ (a) 1 , (b) 2 , (c) 4 . 


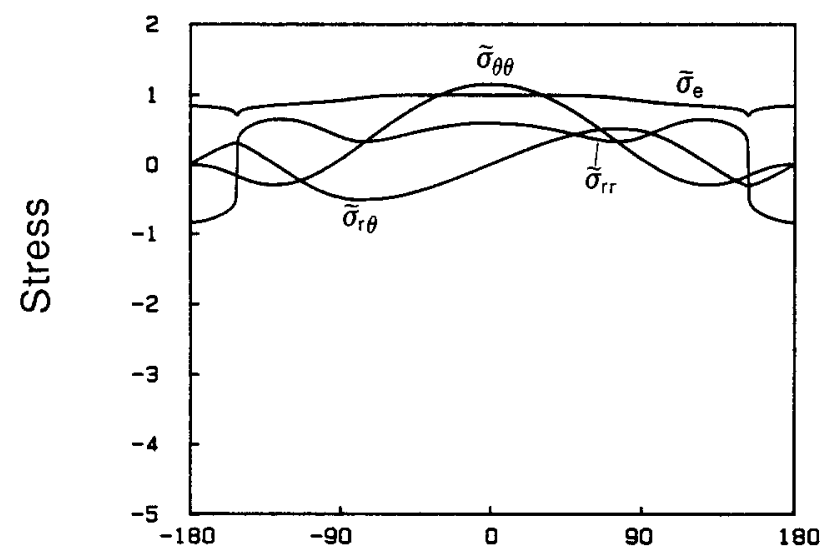

(a)

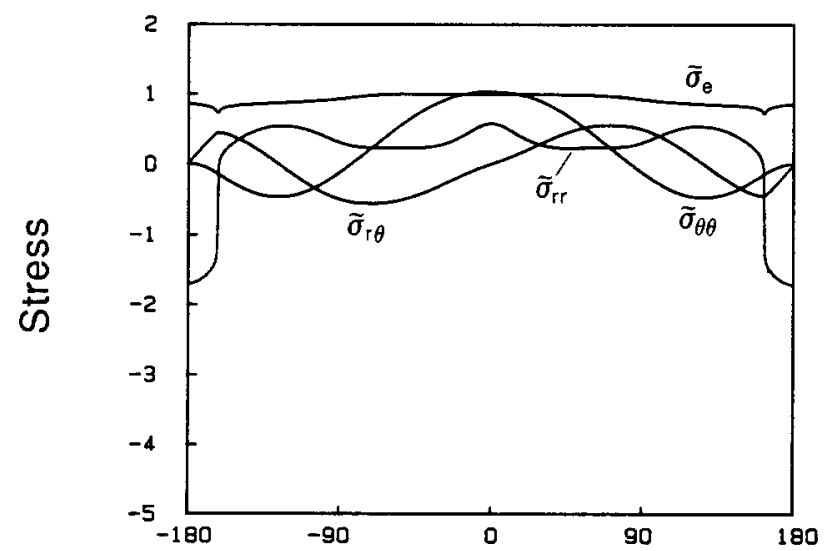

(b)

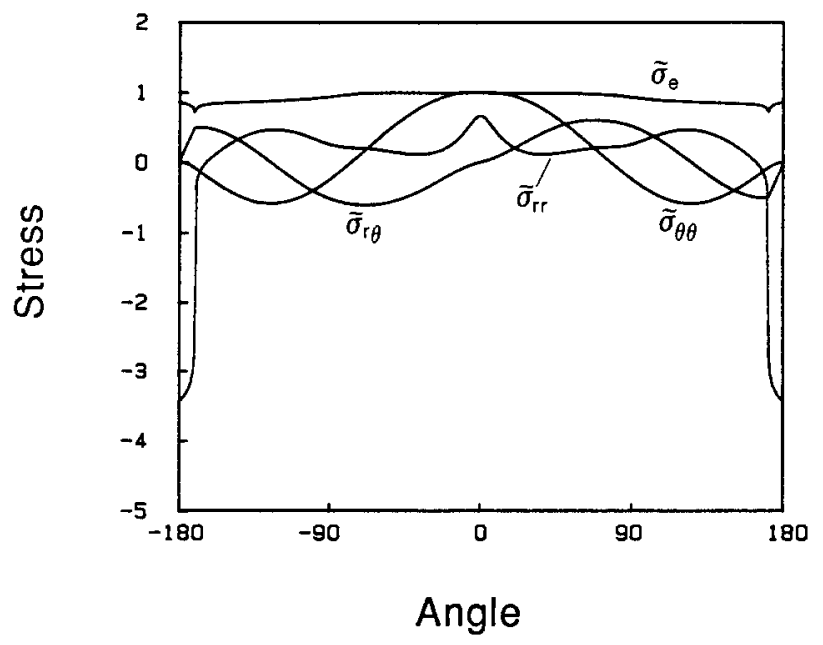

(c)

Fig. 4. Case 1: Angular distributions of the crack-tip stress fields for power-law materials with $n=20$, $Y_{0}=Z_{0}=\sqrt{3} T_{0}$ and $X_{0} / Y_{0}=$ (a) 1 , (b) 2, (c) 4 . 
conditions along the borders of these sectors. Therefore, a discontinuity of $\sigma_{r r}$ and a discontinuity of the derivatives of $\sigma_{r r}$ and $\sigma_{r 0}$ with respect to $\theta$ along the borders of two sectors may exist. Nevertheless, the derivative of $\sigma_{\theta 0}$ with respect to $\theta$ along the borders must be continuous due to the equilibrium equation (2.13) and the continuity of $\sigma_{r 0}$ along the borders. A detailed comparison of the stress fields shown in Fig. 3 with those in Fig. 4 suggests that the perfectly plastic solutions shown in Fig. 3 indeed correspond to the low-hardening limit of the crack-tip fields for the corresponding powerlaw materials.

\subsection{Case 2}

Next, we examine the effects of plastic orthotropy on the crack-tip fields when the strength in the $x_{2}$ direction is increased with respect to the other, i.e., $Y_{0} / X_{0}>1, X_{0}=Z_{0}=\sqrt{3} T_{0}$. Figure 5(a) shows the crack-tip stress field for isotropic materials. Figures 5(b) and 5(c) show the crack-tip stresses for $Y_{0} / X_{0}=2$ and 4 , respectively.

All the crack-tip fields shown in Fig. 5 are type-A fields. A comparison of the stress fields shows that as $Y_{0} / X_{0}$ is increased from 1 to $4, \theta_{C}$ increases from $79.8 \mathrm{deg}$ to $89.1 \mathrm{deg}, \theta_{B}$ decreases from $151.2 \mathrm{deg}$ to $147.2 \mathrm{deg}$, and $\sigma_{00}$ at $\theta=0^{\circ}$ increases from $1.16 \sigma_{e}$ to $4.03 \sigma_{e}$. Since the strength in the $x_{2}$ direction is increased, we expect that the crack-tip normal stress near the $x_{2}$ direction must increase at some angle $\theta$. Figure 5 shows that as the strength in the $x_{2}$ direction is increased, $\sigma_{00}$ near $\theta=0^{\circ}$ and $\sigma_{r r}$ near $\theta=70^{\circ}$ increase. The elevation of hoop stress in front of the crack tip is similar to that of the plane-strain case for $p<1$, where $p$ is the plastic orthotropy parameter defined by Pan and Shih [14]. However, in general, the nature of the plastic sector ahead of the crack tip in plane strain differs from that in plane stress. For example, for isotropic materials in plane stress, there is a centered fan sector in front of the crack tip. This fan sector may induce a large amount of plastic shearing due to a $1 / r$ singularity in strain for elastic perfectly plastic materials [7]. For isotropic materials in plane strain, there is a constant stress sector in front of the crack tip. This constant stress sector induces only a limited amount of plasticity, as shown in the computational studies for elastic perfectly plastic isotropic materials under small-scale yielding conditions by Levy et al. [19], Rice and Tracey [20], Tracey [21], and Parks [22].

The low-hardening crack-tip stress fields $(n=20)$ for power-law materials are shown in Figs. 6(a), 6(b), and 6(c) for the three materials corresponding to Figs. 5(a), 5(b), and 5(c), respectively. The characteristics of the stress fields for each of the corresponding power-law solutions and the perfectly plastic solutions are the same except that the power-law solutions are smoother due to the continuity of the second derivatives of the stresses with respect to $\theta$. Therefore, Figs. 5 and 6 suggest that the perfectly plastic solutions shown in Fig. 5 indeed correspond to the low-hardening limit of the crack-tip fields for the corresponding powerlaw materials.

\subsection{Case 3}

Now we examine the effects of plastic orthotropy on the crack-tip fields when the strength in the $x_{3}$ direction is increased with respect to the others, i.e., $Z_{0} / X_{0}>1, X_{0}=Y_{0}=\sqrt{3} T_{0}$. The transversely isotropic materials that we consider in this case are isotropic in the $x_{1}-x_{2}$ plane and have an orthotropic symmetry axis in the $x_{3}$ direction. Figure 7(a) shows the 


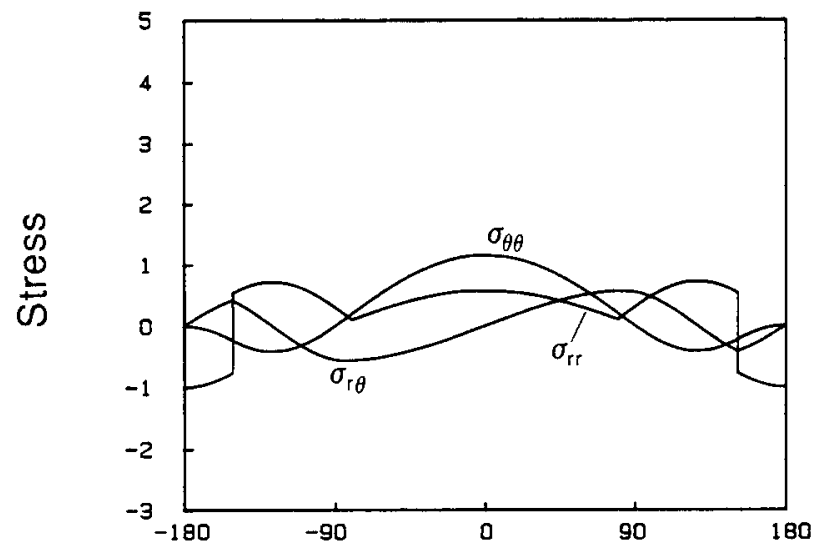

(a)

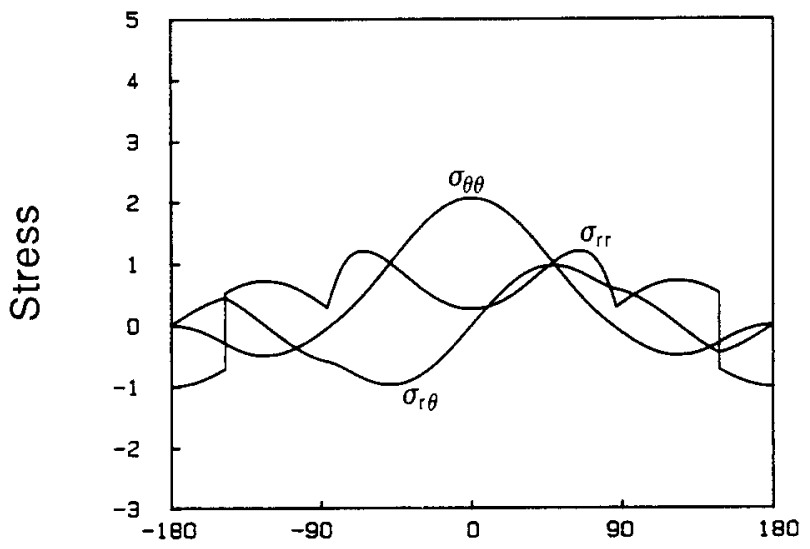

(b)

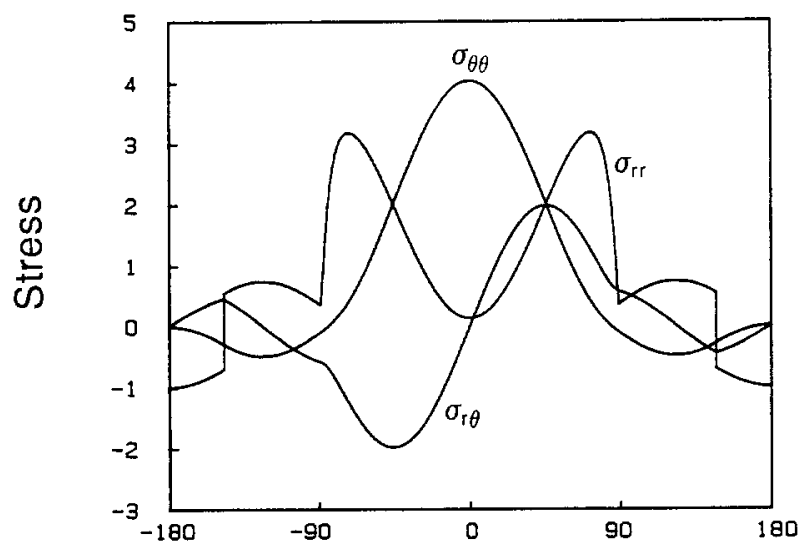

(c)

\section{Angle}

Fig. 5. Case 2: Angular distributions of the perfectly plastic crack-tip stress fields for $Y_{0}=Z_{0}=\sqrt{3} T_{0}$ and $Y_{0} / X_{0}=$ (a) 1 , (b) 2 , (c) 4 . 


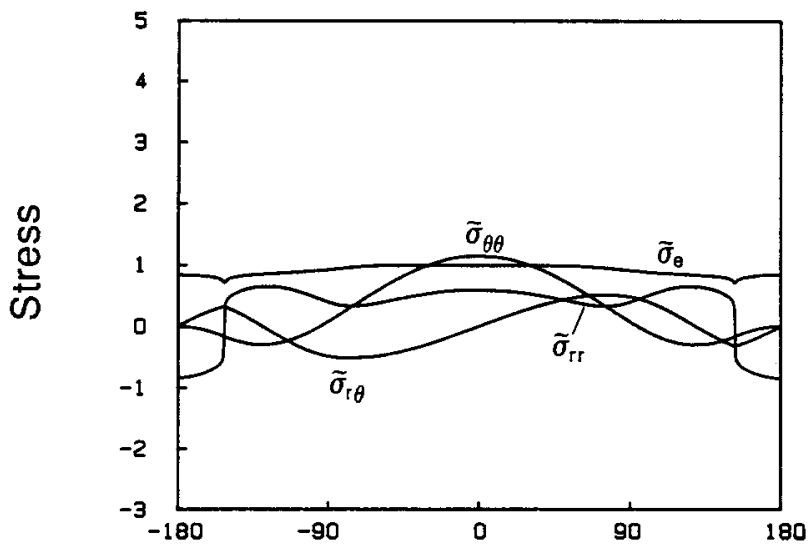

(a)

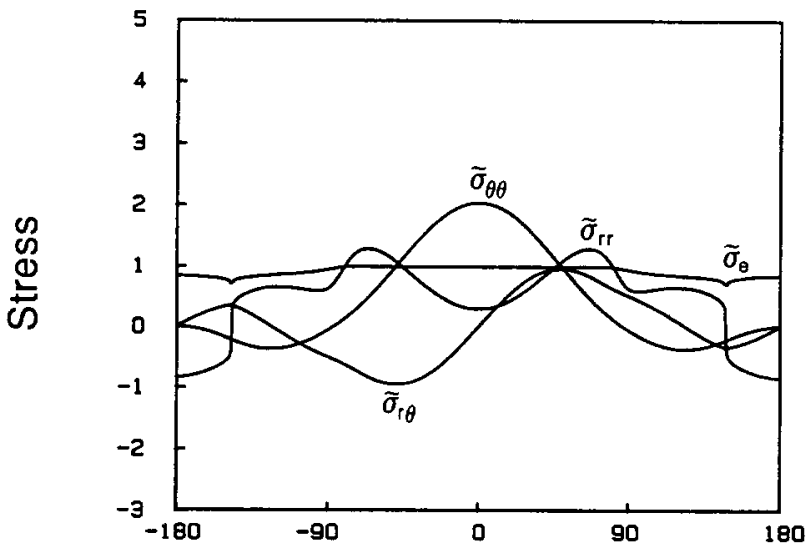

(b)

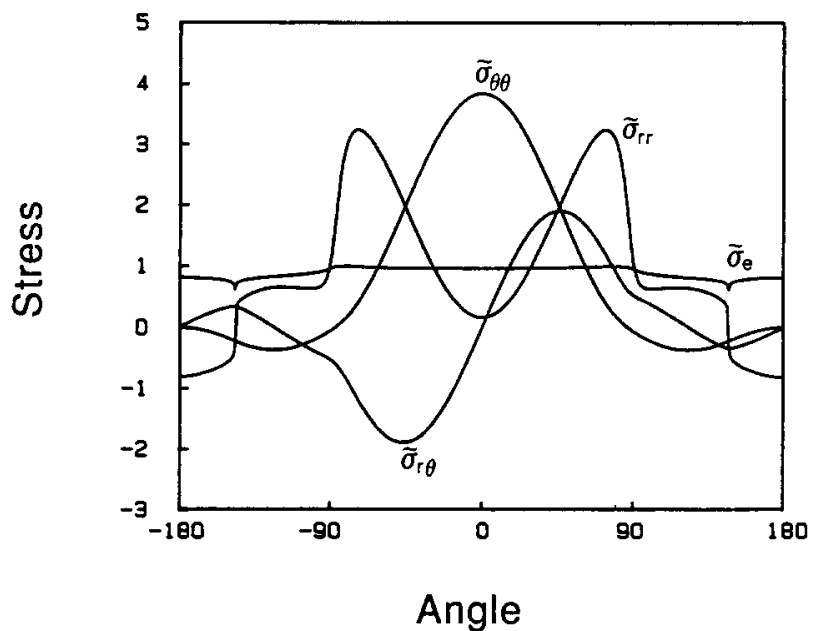

(c)

Fig. 6. Case 2: Angular distributions of the crack-tip stress fields for power-law materials with $n=20, Y_{0}$ $X_{0}=\sqrt{3} T_{0}$ and $Y_{0} / X_{0}=$ (a) 1 , (b) 2 , (c) 4 . 


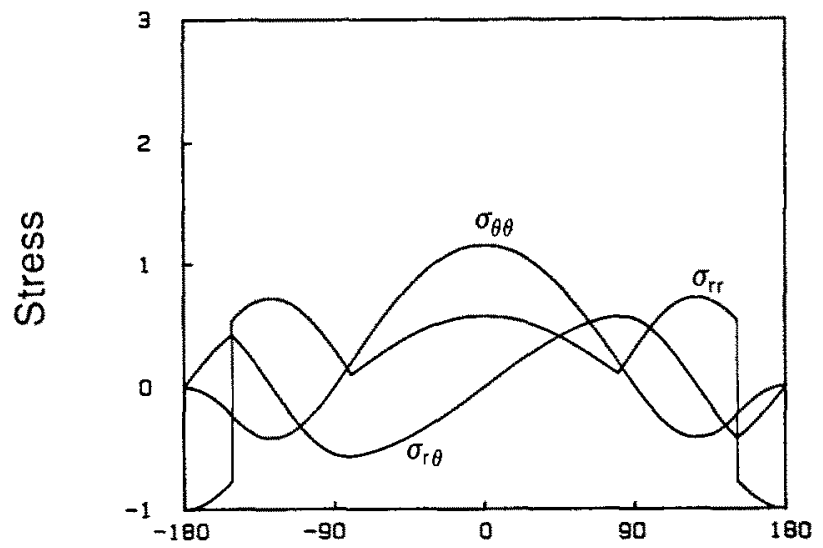

(a)

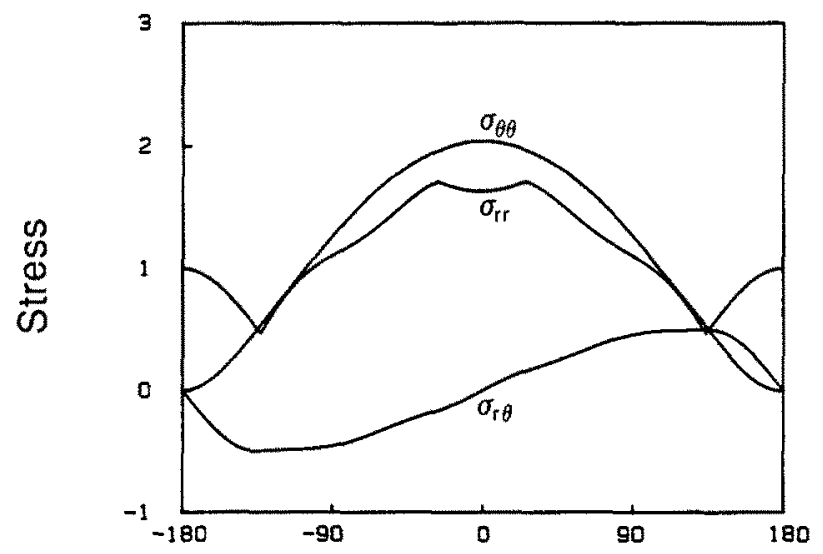

(b)

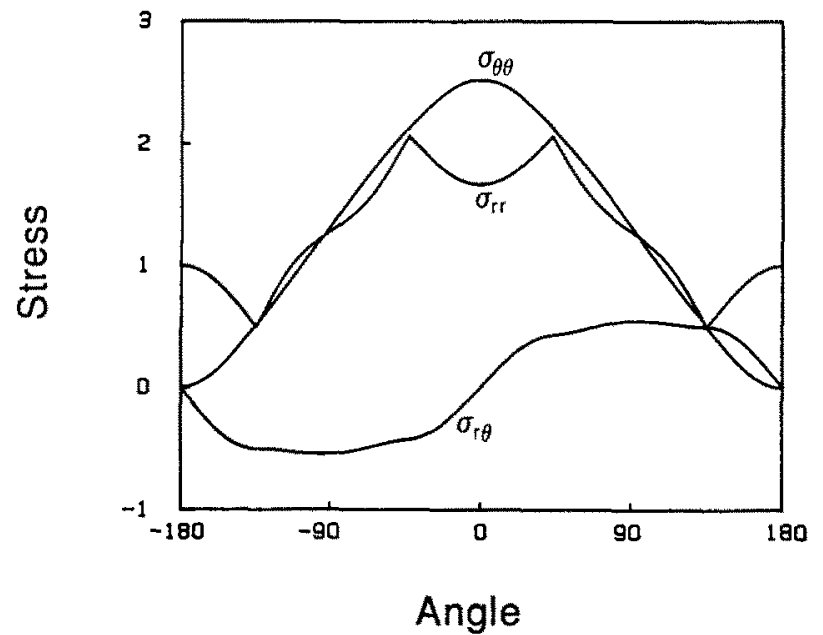

(c)

Fig. 7. Case 3: Angular distributions of the perfectly plastic crack-tip stress fields for $X_{0}=Y_{0}=\sqrt{3} T_{0}$ and $Z_{0} / X_{0}=$ (a) 1 , (b) 2 , (c) 4 . 
crack-tip stress field for isotropic materials. Figures $7(\mathrm{~b})$ and $7(\mathrm{c})$ show the crack-tip stress fields for $Z_{0} / X_{0}=2$ and 4 , respectively.

Note that the crack-tip field for isotropic materials as shown in Fig. 7(a) is a type-A field. The crack-tip fields shown in Figs. 7(b) and 7(c) are type-B fields. As shown in Figs. 7(b) and $7(c)$, we do not have any radial stress discontinuity between the constant stress sectors and the centered fan sector for the type-B fields.

The crack-tip field tends to gain the characteristics of the plane-strain crack-tip field for isotropic materials as $Z_{0} / X_{0}$ becomes large. When we reinforce the strength in the $x_{3}$ direction, we restrain the deformation in the $x_{3}$ direction. Thus we create a plane-strain-like condition in the $x_{3}$ direction regardless of the stress-free conditions in the $x_{3}$ direction in plane stress.

As we increase the ratio $Z_{0} / X_{0}$, the angle of $\theta_{B}$ approaches $180 \mathrm{deg}$ and $\theta_{C}$ approaches 135 $\operatorname{deg}\left(\theta_{B}\right.$ and $\theta_{C}$ are defined in Fig. 2(a) for type-A fields). When the ratio $Z_{0} / X_{0}$ is increased to about 1.4, sector (1) in Fig. 2(a) disappears and a constant stress sector appears in front of the crack tip. The crack-tip field becomes a type-B field, shown in Fig. 2(b).

When the ratio $Z_{0} / X_{0}$ is increased further, $\theta_{\mathrm{D}}$ approaches $45 \mathrm{deg}$ and $\theta_{C}$ approaches 135 deg. As the crack-tip field changes from a type-A field to a type-B field, the border OB between the two constant stress sectors disappears (consequently, the discontinuity of the radial stress $\sigma_{r r}$ of the stress field disappears). The radial stress $\sigma_{r r}$ at $\theta=180^{\circ}$ changes from a negative value to a positive value. This fact is shown in Figs. 7(a), 7(b), and 7(c).

The low-hardening crack-tip stress fields $(n=20)$ for power-law materials are shown in Figs. 8(a), 8(b), and 8(c) for the three materials corresponding to Figs. 7(a), 7(b), and 7(c). The power-law solutions resemble the perfectly plastic solutions for each of the corresponding cases. This suggests that the perfectly plastic solutions shown in Fig. 7 indeed correspond to the low-hardening limit of the crack-tip fields for the corresponding power-law materials. Also, the study of this case suggests that, when the deformation in the out-of-plane direction of a thin sheet is somehow constrained, for example, due to manufacturing processes, the asymptotic field at the tip of a crack in the thin sheet may exhibit the "plane-strain-like" characteristics.

\subsection{Case 4}

Finally, we examine the effects of plastic orthotropy on the crack-tip fields when the in-plane shear strength $T_{0}$ with respect to the $x_{1}$ and $x_{2}$ axes is decreased (or increased) with respect to the tensile strength in the $x_{1}, x_{2}$, and $x_{3}$ directions, i.e., $\sqrt{3} T_{0} / X_{0}<1$ (or $>1$ ), $X_{0}=Y_{0}=Z_{0}$. Because the effective stress is defined with respect to the in-plane shear strength, this case equals the one where the tensile strength in the $x_{1}, x_{2}$, and $x_{3}$ directions is increased (or decreased) with respect to the in-plane shear strength. Figure 9(a) shows the crack-tip stress field for isotropic materials. Figures 9(b) and 9(c) show the normalized crack-tip stress fields $\bar{\sigma}_{i j}$ for $\sqrt{3} T_{0} / X_{0}=1 / 2$ and $1 / 4$, respectively. The normalized crack-tip stress is defined by $\bar{\sigma}_{i j}=\left(\sqrt{3} T_{0} / X_{0}\right) \sigma_{i j}$.

The crack-tip fields for this case are all type-A fields. As shown in Fig. 9, when the shear strength $T_{0}$ is decreased (or the tensile strength in the $x_{1}, x_{2}$, and $x_{3}$ directions is increased), $\theta_{B}$ and $\theta_{C}$ increase; the magnitude of the crack-tip stresses becomes large and increases approximately linearly with the ratio $X_{0} / \sqrt{3} T_{0}$. At $\theta=0^{\circ}$, the hoop stress $\sigma_{\theta \theta}$, which is a very important fracture parameter, also increases. The effect of the elevation of the hoop 


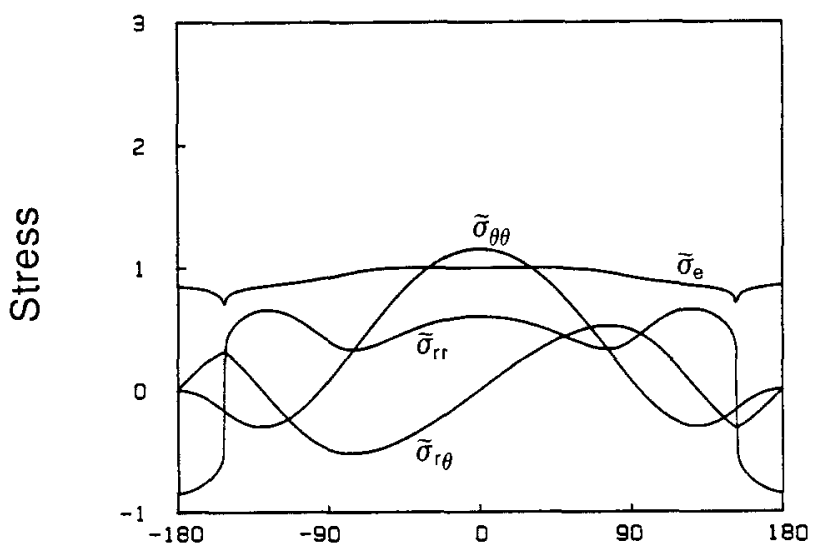

(a)

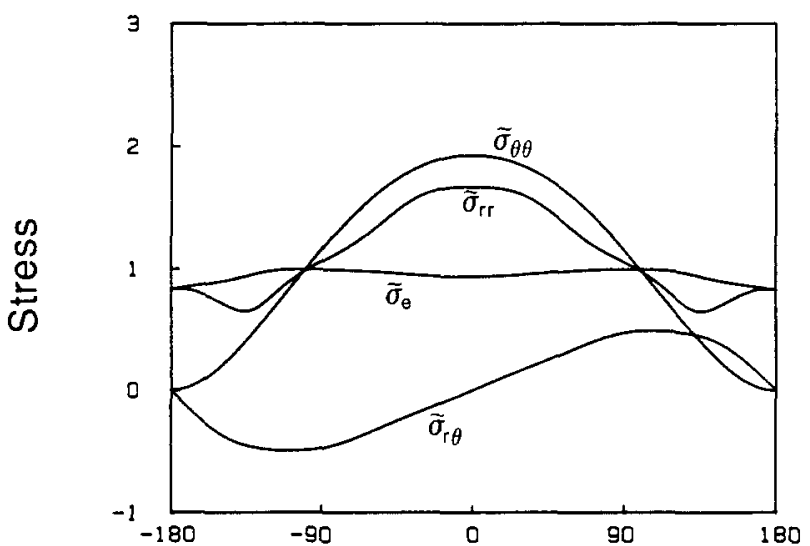

(b)

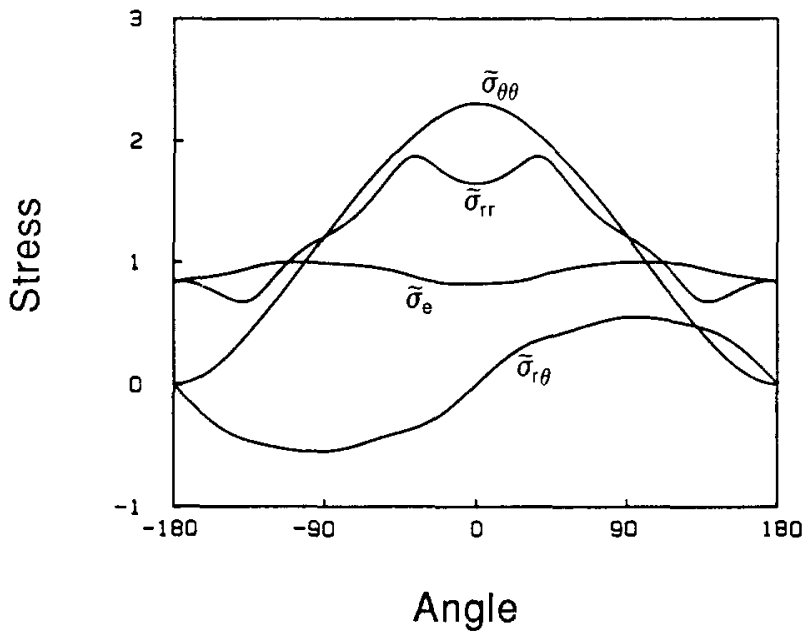

(c)

Fig. 8. Case 3: Angular distributions of the crack-tip stress fields for power-law materials with $n=20$, $X_{0}=Y_{0}=\sqrt{3} T_{0}$ and $Z_{0} / X_{0}=$ (a) 1 , (b) 2 , (c) 4 . 


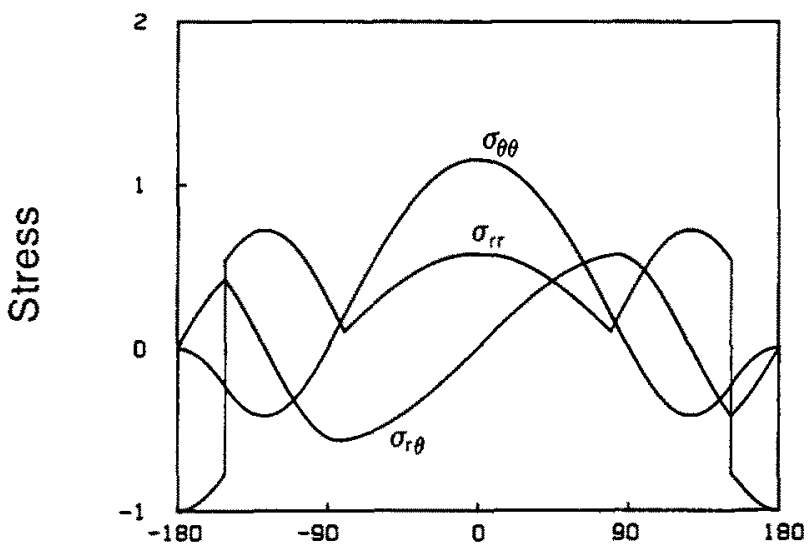

(a)

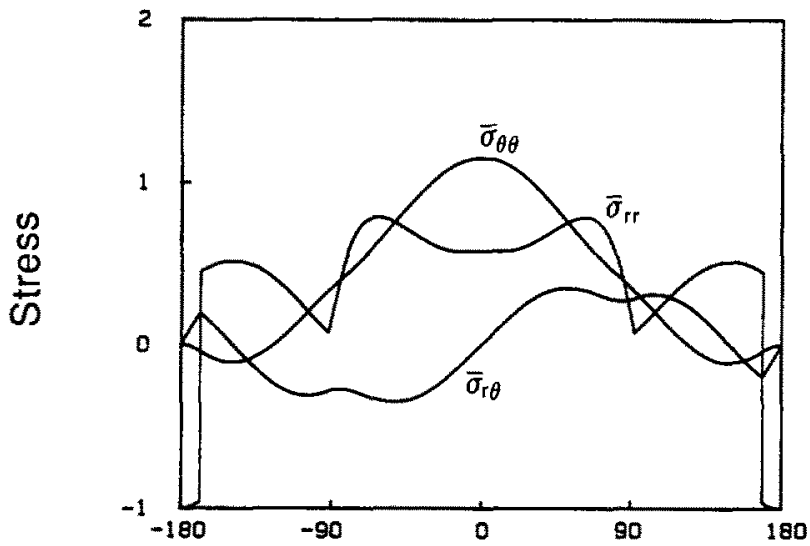

(b)

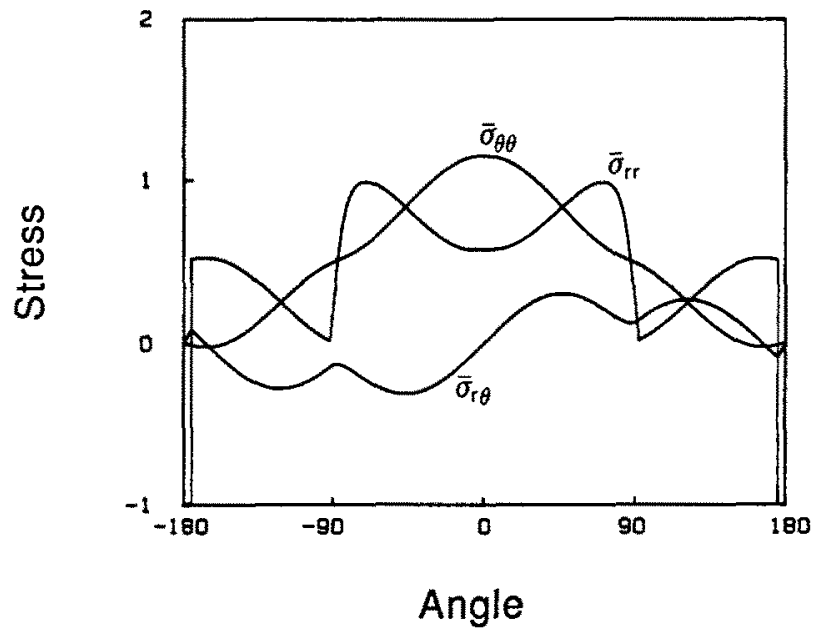

(c)

Fig. 9. Case 4: Angular distributions of the perfectly plastic crack-tip stress fields for $X_{0}=Y_{0}=Z_{0}$ and $\sqrt{3} T_{0} / X_{0}=$ (a) 1, (b) $1 / 2$, (c) $1 / 4$. 
stress in front of the crack tip is the same as that of Case 2 where only the tensile strength in the $x_{2}$ direction is increased with respect to the others.

Figure 10(a) again shows the crack-tip stress field for isotropic materials. Figures $10(\mathrm{~b})$ and $10(\mathrm{c})$ show the normalized crack-tip stress fields $\bar{\sigma}_{i j}$ for $\sqrt{3} T_{0} / X_{0}=2$ and 4 , respectively.

The crack-tip fields in Fig. 10 are still all type-A fields. As shown in Fig. 10, when the in-plane shear strength $T_{0}$ is increased (or the tensile strength in the $x_{1}, x_{2}$, and $x_{3}$ directions is decreased), the magnitude of the stress field becomes small and decreases approximately linearly with the ratio $X_{0} / \sqrt{3} T_{0}$. Also, when the in-plane shear strength $T_{0}$ is increased, both $\theta_{C}$ and $\theta_{B}$ decrease and approach $45 \mathrm{deg}$ and $135 \mathrm{deg}$, respectively. Note that $\theta_{C}$ of the type-A field represents the size of the centered fan secter in front of the crack tip, and the centered fan sector may induce a large amount of plastic shearing at the crack tip [7]. This explains the fact that for Case 4, when the in-plane shear strength $T_{0}$ is increased, the plastic shearing of the asymptotic crack-tip solutions for power-law materials becomes concentrated between $\theta=45^{\circ}$ and $\theta=-45^{\circ}[15]$. The corresponding low-hardening power-law crack-tip stress solutions are very similar to the perfectly plastic solutions and can be found in Pan and Shih [15].

\section{Discussion}

In this paper, we presented the crack-tip fields for perfectly plastic orthotropic materials based on the work of Rice [1]. The construction of the perfectly plastic crack-tip fields is. guided by the corresponding low-hardening stress solutions for power-law materials (Pan and Shih [15]). The results show a close agreement between the perfectly plastic solutions and the low-hardening power-law solutions.

The perfectly plastic solutions we presented here are based on Hill's quadratic anisotropic yield condition. However, the governing equations for the crack-tip field derived by Rice [1] are valid for materials with general anisotropic smooth yield conditions. Therefore, other types of yield conditions such as the non-quadratic yield conditions proposed by Hill [4] or empirical yield conditions can be employed to study the corresponding crack-tip fields. The crack-tip field solutions presented here demonstrate the applicability of the Rice [1] analysis and these solutions indicate the general trends of the effects of plastic anisotropy on the crack-tip fields.

Slip-line theories based on the geometric properties of the yield surfaces in the stress space were introduced by Rice $[17,23]$ for perfectly plastic solids with arbitrary anisotropy under anti-plane and plane-strain conditions. The theories are general enough to treat the yield surfaces' corners and flats, which may exist when crystalline slip is considered as the primary physical process for plastic deformation in crystalline materials. Rice [23] and Rice and Nikolic [24] presented the crack-tip fields for stationary cracks and growing cracks in anisotropic materials such as single crystals under anti-plane shear conditions. Rice [25] also studied the crack-tip fields in single crystals under tensile loading conditions. Pan [16] examined the crack-tip field for anisotropic materials using Hill's quadratic anisotropic yield condition. Note that we can use the governing equations of Rice [1] to investigate the crack-tip field under plane-strain conditions using a plane-strain anisotropic yield condition. However, as shown by Pan [16], the crack-tip fields are easier to construct using the Rice plane-strain slip-line theory [17]. 


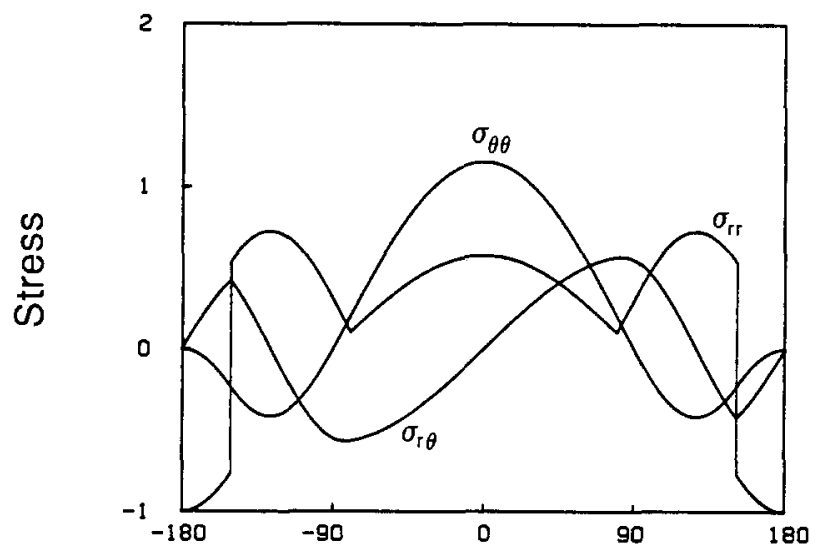

(a)

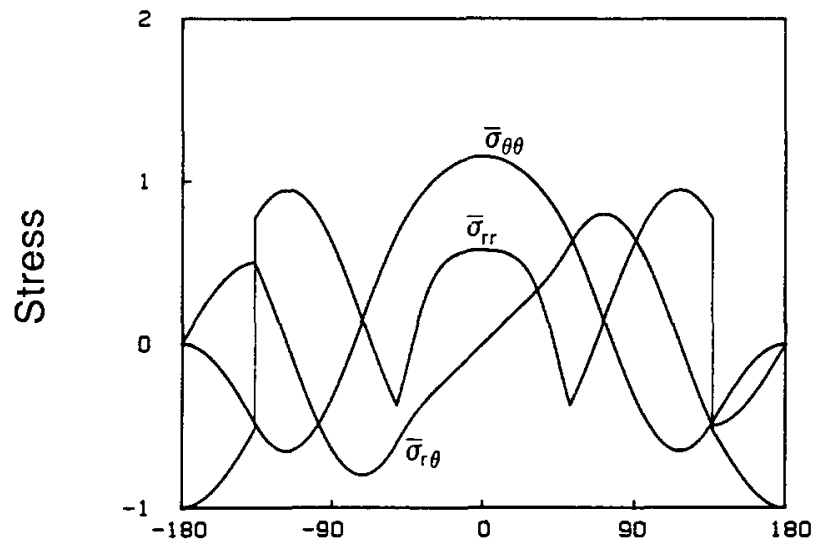

(b)

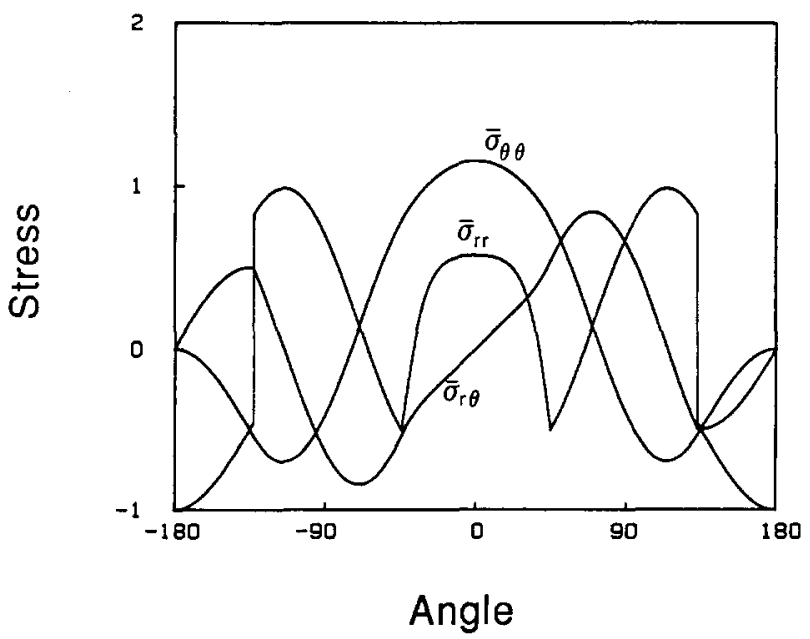

(c)

Fig. 10. Case 4: Angular distributions of the perfectly plastic crack-tip stress fields for $X_{0}=Y_{0}=Z_{0}$ and $\sqrt{3} T_{0} / X_{0}=$ (a) 1 , (b) 2 , (c) 4 . 
Hill's quadratic yield condition in plane-stress has more plastic orthotropy parameters than that in plane-strain, where only one plastic orthotropy parameter is needed to determine a family of the near-tip fields. Under plane-strain mode I conditions, when the orthotropic symmetry axes are aligned with the Cartesian coordinates shown in Fig. 1, the crack-tip slip-line fields for orthotropic materials based on Hill's quadratic yield condition are identical to the Prandtl field for isotropic materials (Pan [16]). In contrast to the plane-strain case, as shown in this study under plane-stress conditions, the size of the plastic sectors in terms of $\theta_{B}$ and $\theta_{C}$ for type-A fields (Fig. 2(a)) and in terms of $\theta_{C}$ and $\theta_{D}$ for type-B fields (Fig. 2(b)) are strong functions of the material orthotropy parameters. This is especially true for Case 3 shown in Fig. 7.

Suppose that the plastic behavior of a ductile composite as an anisotropic continuum can be qualitatively approximated by Hill's anisotropic yield condition adopted here. Using this yield condition, we consider a material where the crack is along the $x_{1}$ direction and reinforcement is along the $x_{2}$ direction. For simplicity, it can be assumed approximately that $Y_{0} / X_{0}>1$ and $X_{0}=Z_{0}=\sqrt{3} T_{0}$ for the materials of this class. The present analysis shows that the crack-tip fields for these materials are type-A fields as shown in Fig. 2(a). Then, according to (3.5) and (3.6), we can solve for $\sigma_{\theta \theta}$ and $\sigma_{r r}$ at $\theta=0^{\circ}$. Note that the shear stress vanishes ahead of the crack tip. The results are $\sigma_{\theta 0} / \sigma_{c}=1 /(F+(H G /(H+G)))^{1 / 2}$ and $\sigma_{r r}=(H /(H+G)) \sigma_{00}$. For $Y_{0} / X_{0}=1,2$, and $4, \sigma_{00} / \sigma_{c}$ is 1.16, 2.06, and 4.03, and $\sigma_{r r} / \sigma_{e}$ is $0.58,0.26$, and 0.12 , respectively. This indicates that as the strength in the $x_{2}$ direction is increased, $\sigma_{\theta 0}$ ahead of the crack tip increases almost linearly with respect to the ratio $Y_{0} / X_{0}$. This information may be useful in the design of ductile composites.

\section{Acknowledgement}

The support of this work by the National Science Foundation under Grant No. MSM8613544 and the Rackham Faculty Grant of the University of Michigan is gratefully acknowledged. Helpful discussion with Professors C.F. Shih and A. Needleman of Brown University is appreciated.

\section{References}

1. J.R. Rice, in Mechanics of Solids: The R. Hill 60 th Anniversary Volume, H.G. Hopkins and M.J. Sewell (eds.), Pergamon Press, Oxford (1982) 539-562.

2. R. Hill, The Mathematical Theory of Plasticity, Oxford University Press, London (1950).

3. R. Hill, Proceedings Royal Society of London, Series A 193 (1948) 281-291.

4. R. Hill, Mathematical Proceedings Cambridge Philosophical Society 85 (1979) $179-191$.

5. J.W. Hutchinson, Journal of the Mechanics and Physics of Solids 16 (1968) 13-31.

6. J.W. Hutchinson, Journal of the Mechanics and Physics of Solids 16 (1968) 337-347.

7. J.R. Rice, Transaction of the ASME, Journal of Applied Mechanics 35 (1968) 379-386.

8. J.R. Rice and G.F. Rosengren, Journal of the Mechanics and Physics of Solids 16 (1968) 1-12.

9. C.F. Shih, "Elastic-Plastic Analysis of Combined Mode Crack Problems," Ph.D. thesis, Harvard University, Cambridge, Mass. (1973).

10. C.F. Shih, in Fracture Analysis, ASTM STP 560 (1974) 187-210.

11. C.F. Shih, "Tables of Hutchinson-Rice-Rosengren Singular Field Quantities," MRL E-147, Material Research Laboratory, Brown University (1983).

12. S. Nemat-Nasser and M. Obata, Mechanics of Materials 3 (1984) 235-247.

13. K. Hayashi, Journal of the Mechanics and Physics of Solids 27 (1979) 163-174. 
14. J. Pan and C.F. Shih, Mechanics of Materials 5 (1986) 299-316.

15. J. Pan and C.F. Shih, International Journal of Fracture 37 (1988) 171-195.

16. J. Pan, Journal of the Mechanics and Physics of Solids 34 (1986) 617-635.

17. J.R. Rice, Journal of the Mechanics and Physics of Solids 21 (1973) 63-74.

18. J.R. Rice, in Fracture, Vol. 2, H. Liebowitz (ed.), Academic Press, New York (1968) 191-311.

19. N. Levy, P.V. Marcal, W.J. Ostergren, and J.R. Rice, International Journal of Fracture Mechanics 7 (1971) 143-156.

20. J.R. Rice and D.M. Tracey, in Numerical and Computer Methods in Structural Mechanics, S.J. Fenves et al. (eds.), Academic Press, New York (1973).

21. D.M. Tracey, Journal of Engineering Materials and Technology 98 (1976) 146-151.

22. D.M. Parks, "Some Problems in Elastic-Plastic Finite Element Analysis of Cracks," Ph.D. thesis, Brown University, Providence, RI (1975).

23. J.R. Rice, Mechanics of Materials 3 (1984) 55-80.

24. J.R. Rice and R. Nikolic, Journal of the Mechanics and Physics of Solids 33 (1985) 595-622.

25. J.R. Rice, "Tensile Crack Tip Fields in Elastic-Ideally Plastic Crystals," Report No. Mech-106, Division of Applied Sciences, Harvard University (July 1987) to be published.

Résumé. On étudie les champs de contraintes planes de mode I à l'extrémité d'une fissure, dans les matériaux orthotropiques parfaitement plastique. L'orthotropie plastique est décrite par la fonction quadratique de plasticité de Hill. On base les constructions des champs de constraintes sur l'analyse générale des constraintes à l'extrémité d'une fissure fournie par Rice pour les matériaux élastiques parfaitement plastiques, que l'on règle par les lois paraboliques caractérisant un faible écrouissage. Lorsque l'on modifie l'orthotropie plastique, il apparaît deux types de champs de contraintes à l'extrémité de la fissure très différents. Le premier comporte un secteur en éventail centré sur le front de fissure, et deux secteurs voisins à contraintes constantes. Le second consiste en une secteur à contrainte au bord de la surface de la fissure, et un secteur en éventail centré sur les deux secteurs à contraintes constantes. Toutes les solutions relatives à une extrémité de fissures parfaitement plastique sont vérifiées par les fonctions paraboliques d'écrouissage faible correspondantes. On discute des tendances générales que suivent les solutions pour les contraintes en extrémité de fissure selon l'orthrotropie plastique, et des implications que comportent ces solutions dans la conception de matériaux composites ductiles. 